\title{
Características morfométricas en dominio discreto para reconocimiento de tumores cerebrales
}

\author{
Angel Carrillo-Bermejo ${ }^{1}$, Nidiyare Hevia-Montiel ${ }^{2}$, Erik Molino-Minero-Re $^{2}$ \\ ${ }^{1}$ Universidad Autónoma de Yucatán, Facultad de Matemáticas, \\ Instituto de Investigaciones en Matemáticas Aplicadas y en Sistemas-Sede Mérida, \\ México \\ ${ }^{2}$ Universidad Nacional Autónoma de México, \\ México \\ angeljcarrillo@gmail.com, nidiyare.hevia@iimas.unam.mx, \\ erik.molino@iimas.unam.mx
}

\begin{abstract}
Resumen. Los tumores cerebrales pueden clasificarse según su agresividad o nivel de malignidad en cuatro grados (I a IV) de menor a mayor agresividad. Los gliomas de bajo grado son tumores vascularizados pero en forma moderada y los gliomas de alto grado presentan áreas con una alta densidad vascular. En la actualidad, las secuencias de imágenes por resonancia magnética se emplean para el diagnóstico y la visualización de la delimitación de las regiones tumorales. En nuestro caso, este trabajo se centra en el análisis morfométrico de tumores cerebrales y estudiar la correlación de la forma del tumor con el grado de malignidad. Proponemos aplicar descriptores morfológicos discretos como el volumen, el área de superficie envolvente, el área de superficie de contacto, la compacidad discreta, la tortuosidad discreta y la relación de volumen. Todos estos descriptores morfométricos se obtienen de regiones binarias segmentadas de las secuencias de resonancia magnética de pacientes con presencia de gliomas. Los resultados muestran una relación inversa entre la compacidad discreta y el grado de malignidad de los gliomas, y una relación directa entre la tortuosidad discreta y el grado de malignidad. La relación de volumen entre la región del tumor y la región del edema es un descriptor útil en la clasificación del tumor.
\end{abstract}

Palabras clave: descriptor de forma, reconocimiento de patrones, compacidad discreta, tortuosidad discreta, carcaterísticas morfológicas en tumores cerebrales.

\section{Discrete-domain Morphometric Descriptors for Brain Tumor Analysis}

Abstract. Brain tumors can be classified according to their agressiveness level into grades I-IV with increasing malignancy. The low-grade gliomas 
are moderately vascularized tumors and high-grade gliomas show areas of high vascular density. At the present time, magnetic resonance imaging sequences are employed for the diagnosis and delimitation of tumor regions. This work focuses on the study of brain tumors through morphometric analysis and their correlation with the degree of malignancy. We propose some discrete morphological descriptors: volume, area of enclosing surface, contact surface area, discrete compactness, discrete tortuosity, and volume ratio obtained from multicontrast magnetic resonance scans of glioma patients. Results show an inverse relationship between discrete compactness and the malignancy grade of gliomas, and a direct relationship between discrete tortuosity and the degree of malignancy. The volume ratio between tumor region and edema region is a descriptor that can be useful for tumor classification.

Keywords: shape descriptor, brain tumor morphometry, discrete compactness, tortuosity.

\section{Introducción}

Este trabajo tiene como objetivo proporcionar algunos descriptores morfométricos discretos, principalmente la compacidad discreta y la tortuosidad discreta, con el interés de analizar la morfometría de tumores cerebrales así como una herramienta computacional para el clínico en el diagnóstico del paciente.

\subsection{Tumores cerebrales}

En la literatura hay varias definiciones sobre un tumor, pero en todos los casos las definiciones coinciden en una definición simple: un tumor es una masa de tejido anormal que crece fuera de control Los tumores cerebrales se originan por el crecimiento de células anormales en los tejidos del cerebro, por ejemplo, las células gliales. Los tumores cerebrales se pueden dividir en categorías diferentes dependiendo de la causa de su origen, la extensión de los tumores, su modo de infiltración y el grado de malignidad [16]. De acuerdo con su origen, los tumores cerebrales se pueden clasificar como tumores cerebrales primarios y tumores cerebrales metastásicos. En el caso de los tumores cerebrales primarios, estos surgen en el cerebro de las células gliales y se pueden definir como glioma. Un tumor cerebral metastásico se origina en otras partes del cuerpo. Los gliomas son los tumores cerebrales más frecuentes en adultos y tienen características similares por su infiltración que puede rodear los tejidos [6].

Los tumores cerebrales también pueden clasificarse por su nivel de agresividad, donde la Organización Mundial de la Salud (OMS), los tumores cerebrales se clasifican en cuatro grados: de I a IV, aumentando su agresividad o malignidad, respectivamente [17]. De esta clasificación de tumores cerebrales primarios, pude considerarse a su vez las cuatro categorías en dos grupos como tumores de bajo grado $(\mathrm{BG})$ y tumores de alto grado $(\mathrm{AG})$, donde el grupo de BG está 
integrado por tumores cerebrales de grado I y II, mientras que el grupo de AG está conformado por tumores cerebrales de grado III y IV. Los tumores de $\mathrm{BG}$, como astrocitoma $\mathrm{u}$ oligodendroglioma, presentan un crecimiento lento y tienen una esperanza de vida de varios años. Estos gliomas de BG son tumores vascularizados de manera moderada. Por otro lado, los gliomas de AG son los tumores más agresivos con una tasa media de supervivencia de un máximo de dos años, estos tumores requieren tratamiento inmediato $[15,13]$. En los tumores de AG su clasificación incluyen el glioblastoma multiforme (GBM), este tipo de tumores son infiltrantes y se extienden a lo largo de los tractos de fibras de materia blanca, crecen vasos anormales y exhiben un núcleo necrótico.

La Figura 1 muestra un corte axial de una resonancia magnética (RM) con un glioma de BG (la imagen del lado izquierdo) y un glioma de AG (lado derecho).
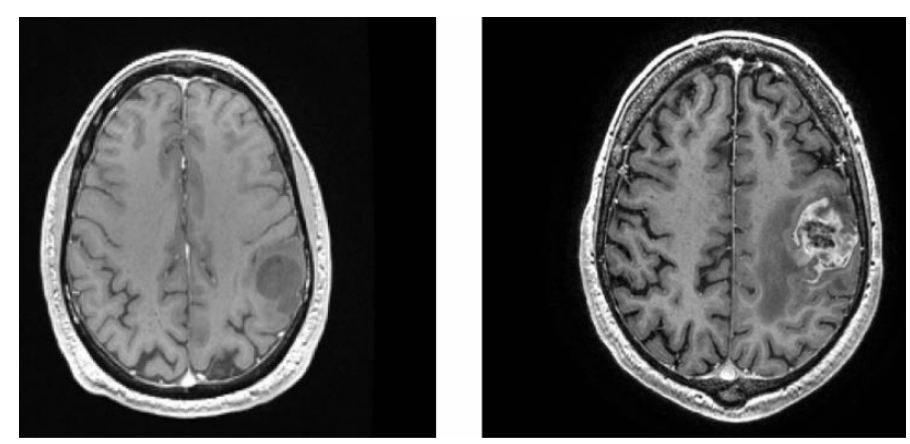

Fig. 1. Ejemplos de cortes axiales a partir de una imagen de RM: paciente con glioma de BG (izquierda) y paciente con glioma de AG (derecha).

\subsection{Neuroimagenología y análisis de gliomas}

La imagenología por resonancia magnética (IRM) es la técnica de adquisición de imágenes médicas más útil para diagnosticar tumores cerebrales. Las secuencias de IRM se usan en el estudio y diagnostico de paciente, planificación del tratamiento y ensayos clínicos [17]. Esta adquisición técnica es una exploración no invasiva y nos permite mostrar imágenes cerebrales en puntos de vista axial, sagital o coronal; estas imágenes se pueden combinar para crear un modelo binario 3D del tumor [16].

Una ventaja de esta técnica de imagen médica es que adquiere una alta resolución en imágenes del cerebro humano, y proporciona un buen contraste del tejido cerebral o contraste del tejido tumoral, dependiendo de las secuencias de IRM adquiridas [8]. Las secuencias de IRM permiten visualizar diferentes contrastes del tejido cerebral de acuerdo a la variación en la excitación y tiempos de repetición en el resonador, por lo que es necesario adquirir diferentes 
secuencias de IRM para el diagnóstico y la segmentación tumoral [1]. A partir de estas secuencias de IRM, podemos adquirir imágenes volumétricas $3 \mathrm{D}$ con un alta resolución [17].

Las secuencias de IRM que normalmente se emplean son $T_{1}$-ponderada IRM $\left(T_{1}\right), T_{1}$-ponderada IRM con realce de contraste $\left(T_{1 C}\right), T_{2}$-IRM $\left(T_{2}\right)$ y $T_{2}$-IRM con fluidos-recuperación de inversión atenuada $\left(T_{2 F L A I R}\right) . T_{1}$ se utiliza porque permite un análisis estructural y anotar los tejidos sanos de manera más fácil. $T_{1 C}$ es la secuencia de IRM donde las fronteras o los contornos de los tumores cerebrales se observan brillantes debido al uso de un agente de contraste (gadolinio-DTPA) que se inyecta en el paciente, este agente de contraste se acumula debido a la alteración de la barrera hematoencefálica en la región tumoral proliferativa; en esto caso de que se pueda distinguir la región tumoral necrótica y la activa. La secuencia de RM $T_{2}$ permite observar la región de edema que rodea el tumor, donde la región del edema parece más brillante que el tumor.

\section{Trabajos previos}

Este trabajo se centra en el análisis morfométrico de tumores cerebrales aplicando descriptores morfométricos en un dominio discreto. El desarrollo de nuevas herramientas y algoritmos matemáticos permite la estimación y cuantificación de algunos aspectos morfológicos de los tumores cerebrales para una mejor comprensión de ellos con la información obtenida de las IRM y su relación con las características biológicas [8].

La segmentación de los tumores cerebrales es crucial para el diagnóstico y el control del crecimiento tumoral, en todos los casos es necesario cuantificar el volumen tumoral con el objetivo de medir e implementar un análisis objetivo [17].

Zacharaki et. al [21] aplicaron métodos de clasificación de patrones para separar dos tipos diferentes de tumores cerebrales, gliomas primarios de metástasis (MET). También propusieron técnicas de reconocimiento de patrones para la clasificación de gliomas. El método de clasificación propuesto combina secuencias convencionales de IRM, este método consistió en extracción de características como la forma del tumor, las características de intensidad, así como la rotación invariante de características de textura. Informaron una precisión, sensibilidad y especificidad para la clasificación de neoplasias de grado bajo y alto de $88 \%$, $85 \%$ y $96 \%$, respectivamente.

Yang et. al [19] presenta la hipótesis del GBM y los MET poseen diferentes atributos morfológicos tridimensionales(3D) basados en sus características físicas. Identificaron una superficie límite distinta entre tejido sano y patológico en la superficie del tumor. Las características morfométricas del índice de forma y la curvatura se calcularon para cada superficie tumoral y se usó para construir un modelo morfométrico de GBM y MET.

Otro trabajo para discriminar entre MET y GBM basado en el análisis morfométrico fue propuesto por Blanchet et. al [2], proponen un análisis de forma como un indicador para discriminar estos 2 tipos de patologías cerebrales 
que permitió la discriminación. La validación cruzada resultó en una clasificación con precisión del $95.8 \%$.

El análisis morfométrico de tumores también incluye clasificación de imágenes basado en la histología tisular, en términos de diferentes componentes, que proporciona una serie de índices de composición tumoral.

Chang et. al [7] proponen dos métodos de clasificación de tejidos a partir de estadísticas morfométricas de diversos ubicaciones y escalas basadas en la coincidencia de pirámide espacial y la coincidencia de pirámide espacial lineal.

Los estudios morfométricos no solo se aplican en tumores cerebrales, Yap et. al [20] propuso una análisis morfométrico cuantitativo del carcinoma hepatocelular, donde el objetivo principal es analizar la relación cuantitativa entre la morfología tumoral y el potencial maligno en los tumores del hígado.

Einenkel et. al [9] mostró una correlación inversa entre la compacidad y el grado de la infiltración tumoral a través de su análisis y cuantificación de la invasión del carcinoma del cuello uterino basado en una reconstrucción tumoral en $3 \mathrm{D}$ del tejido.

En investigaciones previas se presentan resultados preliminares de estudios de morfometría en tumores cerebrales, donde uno de los principales descriptores propuestos es la compacidad discreta. Los resultados preliminares muestran una relación inversa entre la compacidad discreta y el grado de malignidad en tumores primarios [10].

\section{Materiales y métodos}

Proponemos algunos descriptores morfológicos discretos para el análisis de tumores cerebrales y su correlación con el grado de malignidad como en su volumen, área de la superficie envolvente, área de superficie de contacto, compacidad discreta, tortuosidad discreta y relación de volumen se obtienen de multi-contraste RM en pacientes con glioma.

En esta sección presentamos el protocolo de adquisición y la definición de regiones de interés (ROI). Posteriormente, se presentan los descriptores morfométricos propuestos en dominio discreto.

\subsection{Base de datos}

La base de datos consta de 40 IRM multi-contraste de pacientes con glioma, de los cuales 20 pertenecen al grupo de BG (diagnóstico histológico: astrocitoma o oligogoestrocitoma) y 20 al grupo de AG (astrocitoma anaplásico y glioblastoma multiforme tumores). Este conjunto de datos de imágenes se obtuvo a partir de la Multimodal Brain Tumor Segmentation Challenge Organization (BRATS) [12]. Fueron adquiridos en cuatro centros diferentes sobre el curso de varios años, utilizando IMR con diferentes intensidades de campo (1.5T y $3 \mathrm{~T})$ y la implementación de diferente secuencias de imágenes.

Los conjuntos de datos de imágenes de este repositorio comparten los siguientes cuatro IMR: imagen ponderada en $T_{1}$, imagen ponderada en $T_{1}$ con contraste $\left(T_{1 C}\right)$, imagen ponderada en $T_{2}$ y imagen ponderada en $T_{2}$-FLAIR (FLAIR) [14]. 


\subsection{Pre-procesamiento y definición de ROI}

Las imágenes son preprocesadas para homogeneizar los datos. Todos los volúmenes de imágenes de los sujetos fueron co-registrado la $T_{1 C}$ IRM y remuestreado a una resolución isotrópica de $1 \mathrm{~mm}$ en un eje estandarizado de orientación [14]. El conjunto de datos del repositorio de imágenes BRATS también contiene la anotación manual por expertos de cuatro tipos de las regiones [14]: edema, núcleo sin realce, núcleo necrótico y núcleo activo.

El edema se segmentó principalmente a partir de imágenes $T_{2}$, el núcleo activo se segmentó mediante el umbral de intensidades $T_{1 C}$ dentro de la región central del núcleo tumoral, el núcleo necrótico esta definido como las regiones tortuosas y de baja intensidad dentro del núcleo tumoral grueso en $T_{1 C}$, y el núcleo o región del tumor sin realce de contraste se definió como la parte restante del núcleo tumoral grueso menos la región del Núcleo activo y las regiones núcleo necrótico [12]. La Figura 2 muestra las regiones de glioma de BG y de AG.

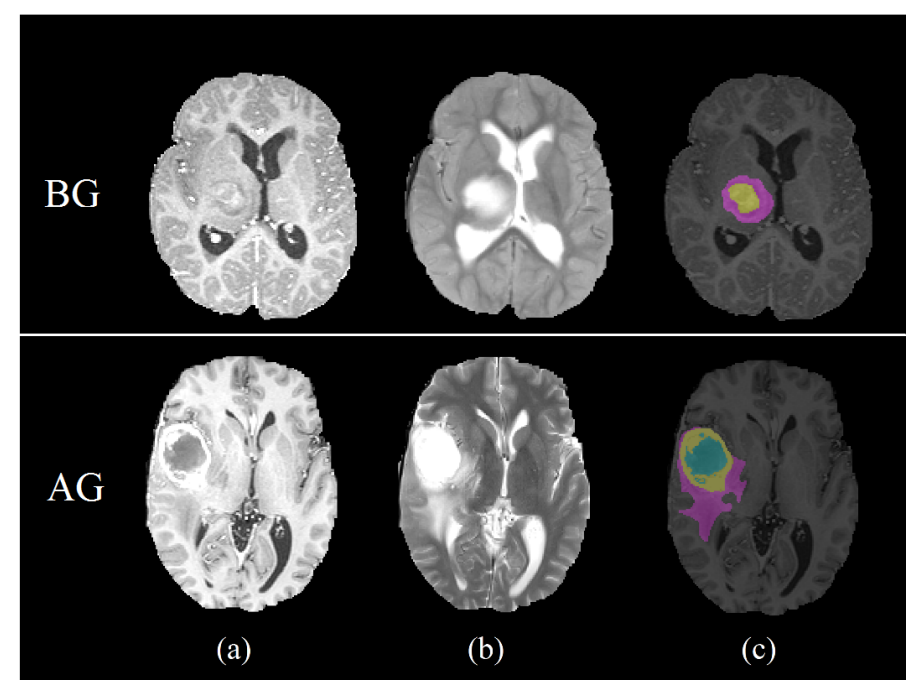

Fig. 2. Ejemplo de vista axial de IRM de BG (primera fila) y AG (segunda fila) pacientes con presencia de glioma. (a) imagen ponderada en $T_{1 C}$ con realce de contraste, (b) imagen ponderada en $T_{2}$ y (c) regiones del tumor cerebral etiquetadas como edema (en púrpura), núcleo activo (en amarillo) y núcleo necrótico (en azul).

Las regiones segmentadas fueron procesadas por el algoritmo de MooreNeighborhood para la detección de bordes con el objetivo de obtener los contornos discretizados 2D para cada región tumoral. La Figura 3 muestra un ejemplo de regiones anotadas (edema, núcleo tumoral, núcleo activo y núcleo necrótico) de un paciente de glioma de AG en la primera fila y sus correspondientes contornos discretos en la segunda fila. 
Características morfométricas en dominio discreto para reconocimiento de tumores cerebrales

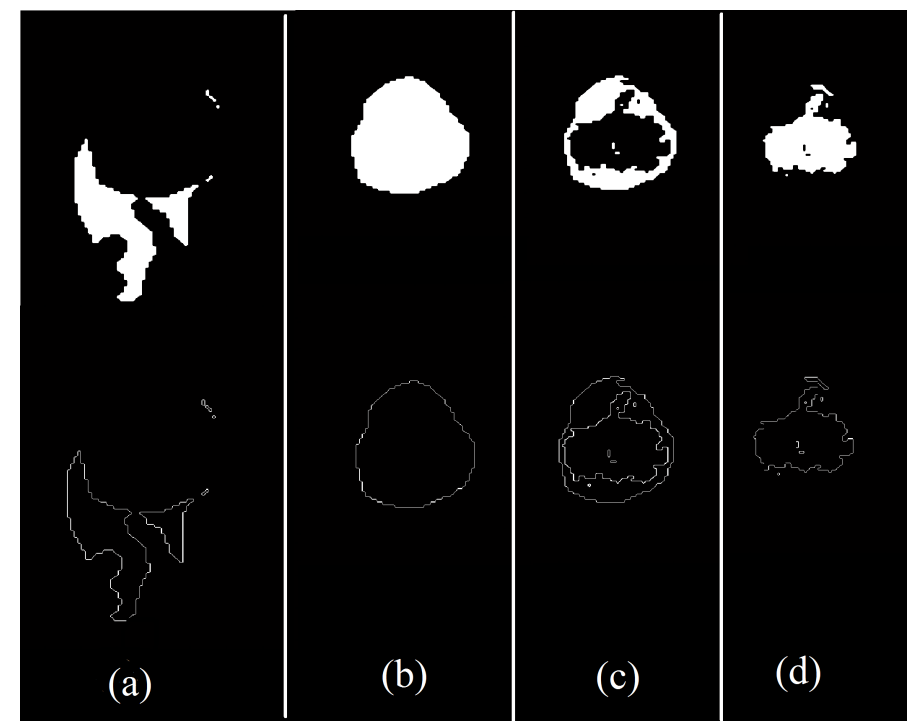

Fig. 3. Se muestran ejemplos de regiones tumorales segmentadas en la primera fila y sus contornos correspondientes detectado en la segunda fila; las regiones tumorales correspondientes son: (a) edema, (b) tumor, (c) núcleo activo y (d) tumor necrótico.

\subsection{Descriptores de forma}

Resumimos las principales características de cada uno de los descriptores morfológicos discretos utilizados para analizar las regiones tumorales, que incluyen el volumen, el área de la superficie envolvente, el área de la superficie de contacto, compacidad discreta, tortuosidad discreta y relación de volumen entre el núcleo tumoral frente al edema.

\subsection{Volumen}

El volumen es una de las características inherentes utilizadas para describir un objeto 3D. Las representaciones volumétricas se utilizan para objetos sólidos rígidos a través de matrices de ocupación espacial. Los objetos discretizados se representan como una matriz 3D de voxeles en el caso de imágenes discretas. El volumen $(V)$ corresponde a la suma de todos los voxeles que componen el objeto $3 \mathrm{D}$, donde el objeto está compuesto de $n$ voxel y cada voxel tiene un volumen igual a uno.

\section{5. Área de la superficie de contacto}

El área de la superficie de contacto $\left(A_{c}\right)$ de un objeto $3 \mathrm{D}$, compuesto por un número finito de $n$ voxels, corresponde a la suma de las áreas de las superficies que son comunes a dos voxeles. 


\section{6. Área de la superficie envolvente}

Considerando el mismo objeto 3D compuesto por un número finito de $n$ voxeles, el área de la superficie envolvente $(A)$ corresponde a la suma de las áreas de los polígonos del plano externo de los voxeles que forman las caras visibles del objeto 3D. En el dominio discreto y de acuerdo con Bribiesca [4], el área de la superficie envolvente $(A)$ se expresa en la ecuación (1), de la siguiente manera:

$$
A=6 a n-2 A_{c},
$$

donde $A_{c}$ es el área de superficie de contacto, $a$ es el área de la cara de un voxel (en este caso $a$ es igual uno, lo que significa que todos los lados de los voxeles son un valor unitario), y $n$ es igual 6 en la Ecuación 1 debido a que indica el número de caras del vóxel, que en este caso es un cubo.

\subsection{Compacidad discreta}

La compacidad $(C)$ es una propiedad intrínseca importante de los objetos, en los objetos 3D esta propiedad relaciona el área de superficie envolvente $(A)$ con el volumen $(V)$, es adimensional y minimizada por una esfera. En el dominio digital, la compacidad discreta $\left(C_{d}\right)$ depende, en gran medida parte de la suma del área de superficie de contacto de los voxeles vecinos para el caso de objetos tridimensionales, por lo tanto, la medida discreta es más robusta y es invariante en la traslación, la rotación y la escala [3,4].

La medida de compacidad discreta $\left(C_{d}\right)$ de un objeto 3D es adimensional y maximizada por un cubo. Si consideramos que el volumen $(V)$ es directamente proporcional al área de superficie de contacto $\left(A_{c}\right)$, entonces proponemos que la medida de compacidad es la relación del área de la superficie envolvente área $(A)$ con respecto al área de superficie de contacto $\left(A_{c}\right)$. El valor mínimo de la $C_{d}$ para objetos $3 \mathrm{D}$ de $n$ voxeles es cero.

\subsection{Curvatura discreta}

La curvatura es el valor absoluto de la tasa de cambio del ángulo de inclinación del línea tangente con respecto a la distancia a lo largo de la curva [18]. La curvatura discreta de una forma discreta en un vértice $Q$ es la linea tangente que forma el ángulo de contingencia $\omega$, que es el cambio de pendiente entre los segmentos continuos de línea recta en ese punto. La Figura 4 (a) ilustra la curvatura continua y discreta, donde el ángulo de la contingencia $\omega$ representa la curvatura discreta los segmentos $A Q$ y $B Q$. La Figura 4 (b) muestra el rango de cambios de pendiente $[0,1)$ y $[0,-1]$, que se consideró en este trabajo [5]. Estos cambios de pendiente es entonces la cadena que define la forma discreta de la curva continua. 


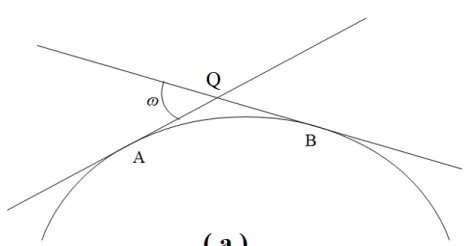

( a )

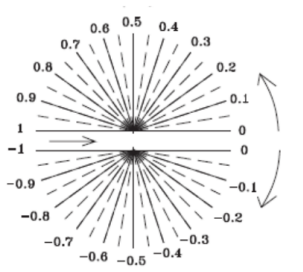

( b )

Fig. 4. (a) Un ejemplo de curvatura continua y discreta, y (b) el rango de cambios de pendiente codificado en $[0,1)$ y $[0,-1)$.

\subsection{Tortuosidad discreta}

La tortuosidad $\tau$ de una curva representada por una cadena definida por Bribiesca [5] como la suma de todos los valores absolutos de los elementos de la cadena, se expresa en la ecuación (2):

$$
\tau=\sum_{i=1}^{n}\left|a_{i}\right| .
$$

La Figura 5 muestra un ejemplo de una curva continua (línea de puntos) y la correspondiente curva discretizada por seis segmentos de línea recta. Por lo tanto, su código cadena es: $-0.33,-0.33,-0.33,0.33,-0.33$ y -0.33 , donde la pendiente acumulada de la curva discreta es igual a -2 y la medida de tortuosidad es igual a 2 .

En nuestro caso, la tortuosidad se mide a partir de los contornos discretos de las regiones tumorales, donde los segmentos de línea recta de estos contornos tienen una longitud de acuerdo con el tamaño y el número de voxeles. En una imagen bidimensional puede haber uno o más contornos, mientras que en una imagen en 3D también puede haber más contornos en cada corte. La $\tau$ discreta para objetos $3 \mathrm{D}$ se define como la suma de todos los valores absolutos de las curvaturas de todos los contornos concatenados presentes en la imagen.

\subsection{Relación volumétrica}

En los gliomas, la expansión tiene lugar dentro del edema circundante, donde las metástasis cerebrales generalmente se asocian con edema peritumoral que puede actuar como un indicador de expansión y tumor más agresivo [11]. Por lo 


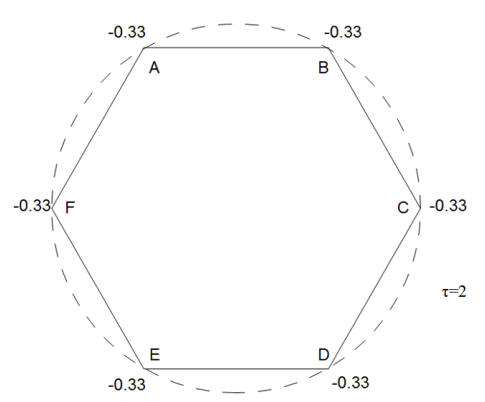

Fig. 5. Un ejemplo de curva continua (línea punteada) y curva discretizada correspondiente a seis segmentos de línea recta, donde la tortuosidad es igual a 2.

tanto, nuestro objetivo fue analizar la relación de volumen $\left(r_{v}\right)$ entre el tumor y edema en gliomas como un descriptor que puede correlacionarse con el grado de malignidad.

\section{Resultados}

Los valores del volumen $(V)$, la superficie del área de $\operatorname{contacto}\left(A_{c}\right)$, área de la superficie envolvente $(A)$, compacidad discreta $\left(C_{d}\right)$, tortuosidad discreta $(\tau)$ y relación volumétrica $\left(r_{v}\right)$, entre región del tumor y edema, se obtuvieron para todas las ROI segmentadas de los 20 pacientes diagnosticados con gliomas de BG (edema y núcleo tumoral) y los 20 pacientes con gliomas de AG (edema, núcleo del tumor, región activa y tumor necrótico). Todos los algoritmos computacionales para cuantificar estos descriptores morfométricos discretos se implementaron en un entorno interactivo para visualización y programación.

La Tabla 1 muestra la tabla de valores medios y la desviación estándar de estos descriptores, obtenidos de todas las regiones segmentadas, para gliomas de BG y AG. Un aumento en el volumen del tumor no es necesariamente relacionado con el grado de malignidad de los gliomas, está más relacionado con el grado de expansión del tumor y la homogeneidad de la superficie periférica de los tumores [10].

Tabla 1. Valores medios y la desviación estándar de edema, núcleo del tumor, núcleo activo y de necrosis para las regiones de gliomas de BG y de AG.

\begin{tabular}{l|c|c|c|c|c|c|c|c}
\hline Descriptor & Edema & Edema & Tumor & Tumor & \multicolumn{2}{c}{ Núcleo Activa Núcleo Activa Necrotica Necrotica } \\
\hline Grado & BG & AG & BG & AG & BG & AG & BG & AG \\
\hline$V\left(\mathrm{~cm}^{3}\right)$ & $7,58 \pm 9,46$ & $15,25 \pm 9,16$ & $6,52 \pm 5,64$ & $10,05 \pm 6,71$ & - & $7,46 \pm 5,86$ & - & $3,70 \pm 3,46$ \\
\hline$A_{c}\left(\mathrm{~cm}^{2}\right)$ & $1083,1 \pm 1373,9$ & $2208,6 \pm 1342,1$ & $951,8 \pm 821,0$ & $1478,3 \pm 991,8$ & - & $1070,1 \pm 850,5$ & - & $524,4 \pm 499,2$ \\
\hline$A\left(\mathrm{~cm}^{2}\right)$ & $107,74 \pm 98,82$ & $157,07 \pm 70,90$ & $52,72 \pm 54,69$ & $58,32 \pm 29,83$ & - & $98,72 \pm 58,60$ & - & $59,83 \pm 45,97$ \\
\hline$C_{d}$ & $0,13 \pm 0,05$ & $0,08 \pm 0,03$ & $0,06 \pm 0,02$ & $0,05 \pm 0,02$ & - & $0,11 \pm 0,05$ & - & $0,18 \pm 0,11$ \\
\hline$T$ & $34,89 \pm 3,72$ & $38,11 \pm 3,01$ & $32,79 \pm 4,62$ & $36,98 \pm 4,10$ & - & $48,23 \pm 8,10$ & - & $44,54 \pm 7,65$ \\
\hline$r_{v}$ & - & - & $1,30 \pm 0,90$ & $0,98 \pm 0,05$ & - & - & - & - \\
\hline
\end{tabular}


Aunque los resultados de la Tabla 1 muestran que el valor del volumen medio para el caso de gliomas de AG es mayor que el valor del volumen medio en el caso de gliomas de BG, esto no necesariamente tiene una correlación con el grado de malignidad de los gliomas. Los resultados muestran una alta variación de los valores del volumen $(V)$, tanto para los gliomas de BG como para los de AG.

Es el mismo caso para el área de la superficie envolvente $(A)$ y la superficie del área de contacto $\left(A_{c}\right)$, donde la variación entre los valores de las áreas presenta una alta desviación. La relación entre el volumen y la superficie del área de contacto es directamente proporcional, como se muestra en la Figura 6, donde se presentan los gráficos de esta relación para los casos de las regiones de edema y núcleo tumoral en todos los pacientes con gliomas de AG y BG de malignidad.
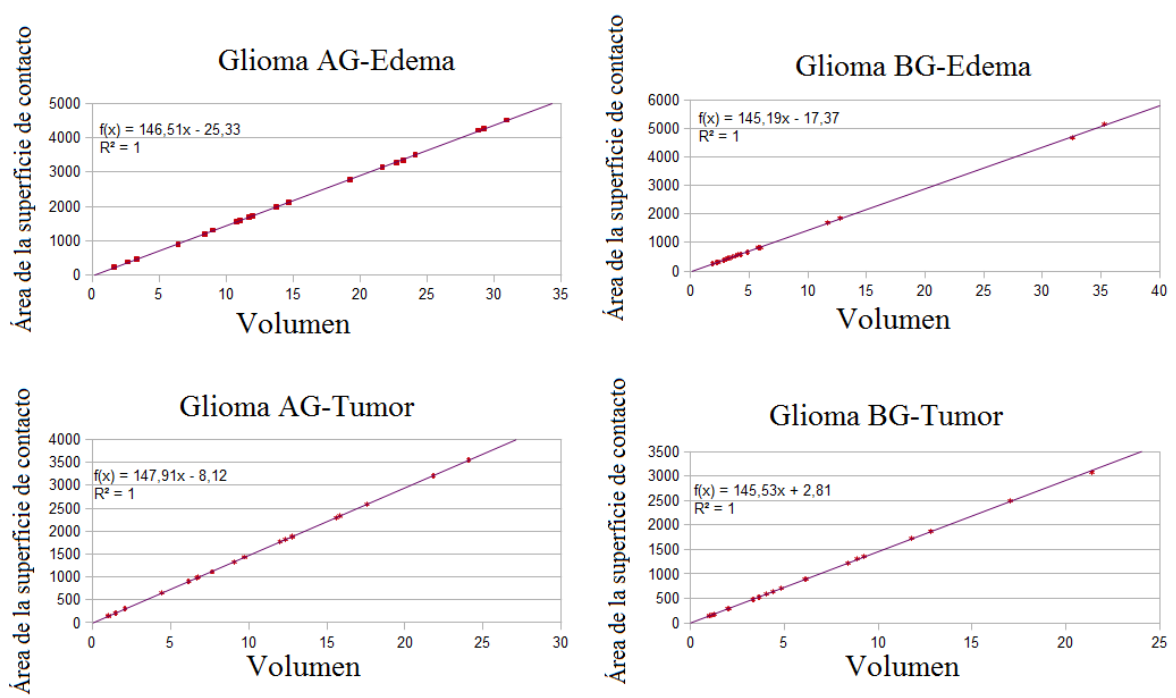

Fig. 6. Relación entre el volumen $(V)$ y la superficie del área de contacto $\left(A_{c}\right)$ en el caso de edema y regiones del núcleo tumoral, tanto para gliomas de BG como de AG. Las gráficas muestran una relación directa, es decir, cuando existe un incremento del volumen, la superficie de contacto se incrementa proporcionalmente.

Como se mencionó anteriormente, en el dominio digital la compacidad discreta $\left(C_{d}\right)$ depende en gran medida de la suma del área de superficie de contacto de los voxeles vecinos, por lo que se definió $C_{d}$ para este estudio como la relación de la superficie envolvente $(A)$ y la superficie del área de contacto $\left(A_{c}\right)$. La $\tau$ discreta se definció como la suma de todos los valores absolutos de las curvaturas de todos los contornos axiales concatenados de las regiones 3D presentes en la imagen.

La Figura 7 y la Figura 8 muestran la comparación de los valores medios de la compacidad y la $\tau$ en el caso de regiones con edema y núcleo tumoral para gliomas de BG y AG respectivamente. 


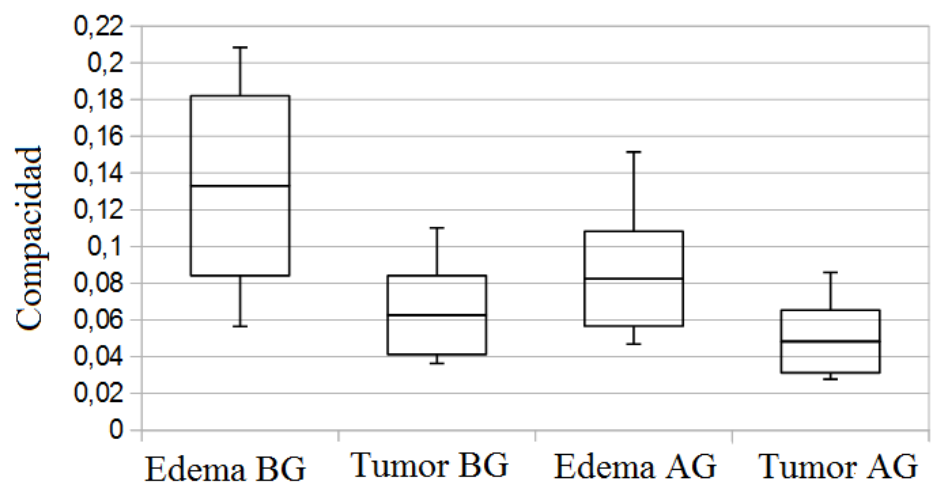

Fig. 7. Representación del espacio de características morfométricas: Compacidad discreta.

Los resultados muestran una correlación inversa entre la compacidad discreta y el grado de malignidad de los gliomas $(\mathrm{r}=-0.3555, \rho=0.0254)$; asi mismo los resultados muestran baja correlación directa entre la tortuosidad versus el grado de malignidad en tumores $(\mathrm{r}=0.4408, \rho=0.0044)$. Los otros descriptores morfométricos como el volumen, el área de la superficie envolvente y el área de contacto mostraron una correlación significativa con el grado de malignidad en tumores cerebrales.

\section{Discusión y conclusión}

Este artículo presenta algunas características morfométricas como volumen, área de superficie envolvente, área de contacto, compacidad, tortuosidad y relación de volúmenes (región del tumor versus la región del edema) para analizar las formas del tumor cerebral y su correlación con el grado de malignidad. Todos los descriptores fueron extraídos de las ROI del edema, núcleo del tumor, núcleo activo y regiones del núcleo necrótico y se implementaron completamente en el dominio discreto para adaptarlos a unidades de voxel en lugar de medidas clásicas. En este enfoque, encontramos que la compacidad discreta y la tortuosidad podrían ser descriptores morfométricos capaces de distinguir entre gliomas de BG y AG.

La Figura 7 y la Figura 8 muestran que los gliomas de BG tienen ligeramente un mayor valor de compacidad que los gliomas de AG, pero un menor valor de tortuosidad en comparación con un glioma de AG. Sin embargo, la diferencia de los valores discretos de compacidad y tortuosidad, entre los gliomas de BG y AG son bajos. Cabe señalar que la segmentación tumoral presenta algunas limitaciones asociadas con los métodos de pre-procesamiento y segmentación de las secuencias de IRM, debido a que las imágenes fueron co-registradas y remues- 


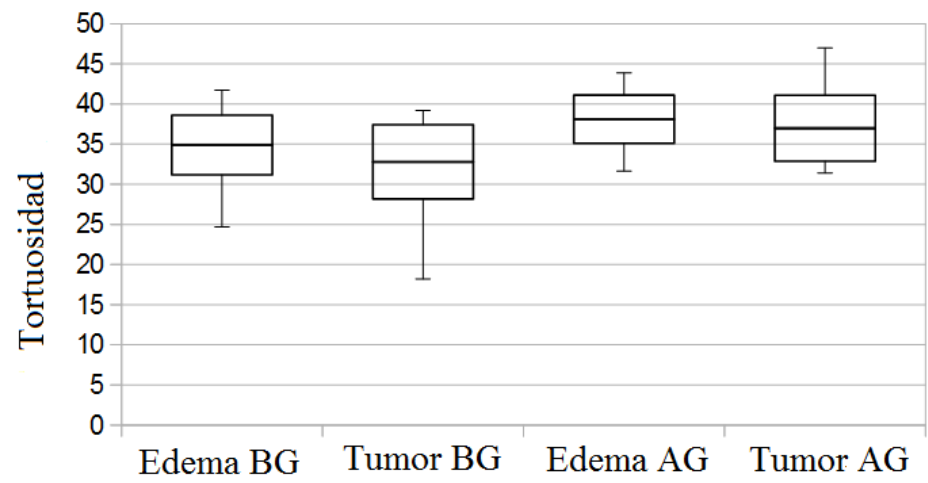

Fig. 8. Representación del espacio de características morfométricas: tortuosidad discreta.

treadas a 1mm de resolución isotrópica en una orientación axial estandarizada, que podría introducir algo de suavizado en la superficie del tumor.

Del mismo modo, las secuencias de IRM se anotaron mediante el delineamiento de las regiones tumorales en cada tres cortes axiales, interpolando la segmentación así como mediante el uso de operadores morfológicos y técnicas de crecimiento de región, de modo que en este caso las regiones tumorales no corresponden en su totalidad con la estructura del tumor real. Dado que los valores discretos de compacidad y tortuosidad son sensibles a la superficie del tumor, es necesario obtener estos descriptores morfométricos discretos de una manera confiable y robusta de la región segmentada y en consecuencia, los coeficientes de correlación del grado de malignidad tumoral con la compacidad discreta y tortuosidad podría mejorarse.

Agradecimientos. Agradecimiento especial al Consejo Nacional de Ciencia y Tecnología (CONACYT) por su apoyo como becario de la maestría y a la Universidad Autónoma Nacional de México (UNAM) por el apoyo parcial en la investigación realizada gracias al Programa UNAM-PAPIIT: IA102918.

\section{Referencias}

1. Imaging of brain tumors with histological correlations. American Journal of Neuroradiology 24(9), 1921-1921 (2003), http://www.ajnr.org/content/24/9/1921

2. Blanchet, L., Krooshof, P., Postma, G., Idema, A., Goraj, B., Heerschap, A., Buydens, L.: Discrimination between metastasis and glioblastoma multiforme based on morphometric analysis of MR images. American Journal of Neuroradiology 32(1), 67-73 (01 2011), http://www.ajnr.org/content/early/2010/11/04/ajnr.A2269

3. Bribiesca, E.: A measure of compactness for 3D shapes 40, 1275-1284 (11 2000) 
4. Bribiesca, E.: An easy measure of compactness for $2 \mathrm{D}$ and 3D shapes. Pattern Recognition 41(2), $543 \quad-\quad 554 \quad$ (2008), http://www.sciencedirect.com/science/article/pii/S003132030700324X

5. Bribiesca, E.: A measure of tortuosity based on chain coding. Pattern Recognition 46(3), $716 \quad-\quad 724 \quad$ (2013), http://www.sciencedirect.com/science/article/pii/S0031320312004232

6. C. Holland, E.: Progenitor cells and glioma formation 14, 683-8 (01 2002)

7. Chang, H., Borowsky, A., Spellman, P., Parvin, B.: Classification of tumor histology via morphometric context. In: 2013 IEEE Conference on Computer Vision and Pattern Recognition. pp. 2203-2210 (June 2013)

8. Clarke, J.L., Chang, S.M.: Neuroimaging: diagnosis and response assessment in glioblastoma. Cancer journal 18 1, 26-31 (2012)

9. Einenkel, J., Braumann, U.D., Horn, L.C., Pannicke, N., Kuska, J.P., Schütz, A., Hentschel, B., Höckel, M.: Evaluation of the invasion front pattern of squamous cell cervical carcinoma by measuring classical and discrete compactness 31, 428-35 (10 2007)

10. Hevia, N., I. Rodriguez-Perez, P., Lamothe-Molina, P., Arellano-Reynoso, A., Bribiesca, E., A. Alegria-Loyola, M.: Neuromorphometry of primary brain tumors by magnetic resonance imaging 2, 024503 (05 2015)

11. Kerschbaumer, J., Bauer, M., Popovscaia, M., Grams, A.E., Thomé, C., Freyschlag, C.F.: Correlation of tumor and peritumoral edema volumes with survival in patients with cerebral metastases. Anticancer Research 37(2), 871-875 (2017), http://ar.iiarjournals.org/content/37/2/871.abstract

12. Kistler, M., Bonaretti, S., Pfahrer, M., Niklaus, R., Büchler, P.: The virtual skeleton database: An open access repository for biomedical research and collaboration. J Med Internet Res 15(11), e245 (Nov 2013), http://www.jmir.org/2013/11/e245/

13. Louis, D.N., Perry, A., Reifenberger, G., von Deimling, A., Figarella-Branger, D., Cavenee, W.K., Ohgaki, H., Wiestler, O.D., Kleihues, P., Ellison, D.W.: The 2016 World Health Organization classification of tumors of the central nervous system: a summary. Acta Neuropathologica 131(6), 803-820 (Jun 2016), https://doi.org/10.1007/s00401-016-1545-1

14. Menze, B., Jakab, A., Bauer, S., Kalpathy-Cramer, J., Farahaniy, K., Kirby, J., Burren, Y., Porz, N., Slotboomy, J., Wiest, R., Lancziy, L., Gerstnery, E., Webery, M.A., Arbel, T., B. Avants, B., Ayache, N., Buendia, P., Collins, L., Cordier, N., Van Leemput, K.: The multimodal brain tumor image segmentation benchmark (BRATS). IEEE Transactions on Medical Imaging 34(10), 1993-2024 (Oct 2015)

15. Okamoto, Y., Di Patre, P.L., Burkhard, C., Horstmann, S., Jourde, B., Fahey, M., Schüler, D., Probst-Hensch, N.M., Yasargil, M.G., Yonekawa, Y., Lütolf, U.M., Kleihues, P., Ohgaki, H.: Population-based study on incidence, survival rates, and genetic alterations of low-grade diffuse astrocytomas and oligodendrogliomas. Acta Neuropathologica 108(1), 49-56 (Jul 2004), https://doi.org/10.1007/s00401-0040861-z

16. Roy, S., Bandyopadhyay, S.: Detection and quantification of brain tumor from MRI of brain and it's symmetric analysis 2, 477-483 (06 2012)

17. Thivya Roopini, I., Vasanthi, M., Rajinikanth, V., Rekha, M., Sangeetha, M.: Segmentation of tumor from brain MRI using fuzzy entropy and distance regularised level set. In: Nandi, A.K., Sujatha, N., Menaka, R., Alex, J.S.R. (eds.) Computational Signal Processing and Analysis. pp. 297-304. Springer Singapore, Singapore (2018)

18. Towler, K.: Mathematics dictionary: algorithms and rules in mathematics for secondary school students. North Ryde, N.S.W.: McGraw-Hill Australia (2004) 
Características morfométricas en dominio discreto para reconocimiento de tumores cerebrales

19. Yang, G., Jones, T., Howe, F., R Barrick, T.: Morphometric model for discrimination between glioblastoma multiforme and solitary metastasis using threedimensional shape analysis 75(6), 2505-2516 (07 2015)

20. Yap, F., T Bui, J., Grace Knuttinen, M., M Walzer, N., Cotler, S., A Owens, C., L Berkes, J., C Gaba, R.: Quantitative morphometric analysis of hepatocellular carcinoma: Development of a programmed algorithm and preliminary application 19, 97-105 (11 2012)

21. Zacharaki, E.I., Wang, S., Chawla, S., Yoo, D.S., Wolf, R., Melhem, E.R., Davatzikos, C.: Classification of brain tumor type and grade using MRI texture and shape in a machine learning scheme. Magnetic Resonance in Medicine 62(6), 1609-1618 (2009), https://onlinelibrary.wiley.com/doi/abs/10.1002/mrm.22147 